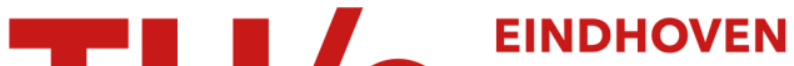 UNIVERSITY OF TECHNOLOGY
}

\section{Model order reduction for nonlinear IC models with POD}

Citation for published version (APA):

Verhoeven, A., Striebel, M., \& Maten, ter, E. J. W. (2009). Model order reduction for nonlinear IC models with $P O D$. (CASA-report; Vol. 0921). Technische Universiteit Eindhoven.

Document status and date:

Published: 01/01/2009

\section{Document Version:}

Publisher's PDF, also known as Version of Record (includes final page, issue and volume numbers)

\section{Please check the document version of this publication:}

- A submitted manuscript is the version of the article upon submission and before peer-review. There can be important differences between the submitted version and the official published version of record. People interested in the research are advised to contact the author for the final version of the publication, or visit the $\mathrm{DOI}$ to the publisher's website.

- The final author version and the galley proof are versions of the publication after peer review.

- The final published version features the final layout of the paper including the volume, issue and page numbers.

Link to publication

\section{General rights}

Copyright and moral rights for the publications made accessible in the public portal are retained by the authors and/or other copyright owners and it is a condition of accessing publications that users recognise and abide by the legal requirements associated with these rights.

- Users may download and print one copy of any publication from the public portal for the purpose of private study or research.

- You may not further distribute the material or use it for any profit-making activity or commercial gain

- You may freely distribute the URL identifying the publication in the public portal.

If the publication is distributed under the terms of Article 25fa of the Dutch Copyright Act, indicated by the "Taverne" license above, please follow below link for the End User Agreement:

www.tue.nl/taverne

Take down policy

If you believe that this document breaches copyright please contact us at:

openaccess@tue.nl

providing details and we will investigate your claim. 


\section{EINDHOVEN UNIVERSITY OF TECHNOLOGY}

Department of Mathematics and Computer Science

\section{CASA-Report 09-21}

June 2009

\section{Model order reduction for nonlinear \\ IC models with POD}

\section{by}

A. Verhoeven, M. Striebel, E.J.W. ter Maten

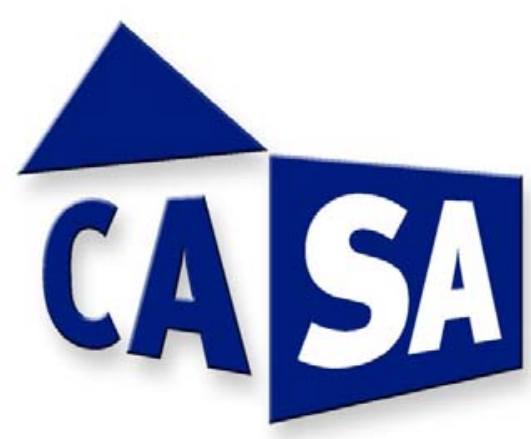

Centre for Analysis, Scientific computing and Applications

Department of Mathematics and Computer Science

Eindhoven University of Technology

P.O. Box 513

5600 MB Eindhoven, The Netherlands

ISSN: 0926-4507 



\title{
Model Order Reduction for Nonlinear IC Models with POD
}

\author{
Arie Verhoeven ${ }^{1}$, Michael Striebel ${ }^{2}$, and E. Jan W. ter Maten ${ }^{3}$
}

\begin{abstract}
Due to refined modelling of semiconductor devices and increasing packing densities, reduced order modelling of large nonlinear systems is of great importance in the design of integrated circuits (ICs). Despite the linear case, methodologies for nonlinear problems are only beginning to develop. The most practical approaches rely either on linearisation, making techniques from linear model order reduction applicable, or on proper orthogonal decomposition (POD), preserving the nonlinear characteristic. In this paper we focus on POD. We demonstrate the missing point estimation and propose a new adaption of POD to reduce both dimension of the problem under consideration and cost for evaluating the full nonlinear system.
\end{abstract}

\section{Introduction}

The dynamics of electrical circuits at time $t$ can be generally described by a nonlinear, first order, differential-algebraic equation (DAE) system of the form:

$$
\left\{\begin{aligned}
\frac{d}{d t}[\mathbf{q}(\mathbf{x}(t))]+\mathbf{j}(\mathbf{x}(t))+\mathbf{B u}(t) & =\mathbf{0} \\
\mathbf{y}(t) & =\mathbf{C}^{T} \mathbf{x}(t)
\end{aligned}\right.
$$

where $\mathbf{x}(t) \in \mathbb{R}^{n}$ represents the unknown vector of circuit variables at time $t \in \mathbb{R}$; $\mathbf{q}, \mathbf{j}: \mathbb{R}^{n} \rightarrow \mathbb{R}^{n}$ describe the contribution of reactive and nonreactive elements, respectively; B $\in \mathbb{R}^{n \times m}$ distributes the input exitation $u: \mathbb{R} \rightarrow \mathbb{R}^{m}$ and $\mathbf{C} \in \mathbb{R}^{n \times q}$ maps

Arie Verhoeven

VORtech Computing, Delft,The Netherlands, e-mail: Arie.Verhoeven@na-net . ornl .gov

E. Jan W. ter Maten

NXP Semiconductors, Eindhoven, The Netherlands, e-mail: Jan.ter.Maten@ nxp.com

Michael Striebel

Chemnitz University of Technology, Chemnitz, Germany, e-mail: Michael.Striebel@

mathematik.tu-chemnitz.de 
the state $\mathbf{x}$ to the system response $\mathbf{y}(t) \in \mathbb{R}^{q}$. In circuit design the input $\mathbf{u}$ and the output $\mathbf{y}$ are terminal voltages and terminal currents, respectively, or vice versa. Therefore, we assume that they are linearly injected and extracted, respectively.

The dimension $n$ of the unknown vector $\mathbf{x}(t)$ is of the order of the number of elements in the circuit, which can easily reach hundreds of millions. Therefore, one may solve the network equations (1) by means of computer algebra in an unreasonable amount of time only.

Model order reduction (MOR) aims to replace the original model (1) by a system

$$
\left\{\begin{aligned}
\frac{d}{d t}[\tilde{\mathbf{q}}(\mathbf{z}(t))]+\tilde{\mathbf{j}}(\mathbf{z}(t))+\tilde{\mathbf{B}} \mathbf{u}(t) & =\mathbf{0} \\
\tilde{\mathbf{y}}(t) & =\tilde{\mathbf{C}}^{T} \tilde{\mathbf{x}}(t),
\end{aligned}\right.
$$

with $\mathbf{z}(t) \in \mathbb{R}^{r} ; \tilde{\mathbf{q}}, \tilde{\mathbf{j}}: \mathbb{R}^{r} \rightarrow \mathbb{R}^{r}$ and $\tilde{\mathbf{B}} \in \mathbb{R}^{r \times m}$ and $\tilde{\mathbf{C}} \in \mathbb{R}^{r \times q}$, which can compute a system response $\tilde{\mathbf{y}}(t) \in \mathbb{R}^{q}$ that is sufficiently close to $\mathbf{y}(t)$ given the same input signal $\mathbf{u}(t)$, but in much less time.

\section{Linear versus nonlinear model order reduction}

So far most research effort was spent on developing and analysing MOR techniques suitable for linear problems. For an overview on these methods we refer to [1].

Research on and applications of MOR for nonlinear problems can still be found less frequent. Some approaches like balanced truncation for nonlinear problems [2, 3 ] are accurate but yet hard to be applied in an industrial context. Others are only feasible for weakly nonlinear dependencies. Then again, when trying to transfer approaches from linear MOR, especially projection based methods, fundamental differences emerge.

To see this, first consider a linear problem of the form

$$
\mathbf{E} \frac{d}{d t} \mathbf{x}(t)+\mathbf{A} \mathbf{x}(t)+\mathbf{B u}(t)=0, \quad \text { with } \mathbf{E}, \mathbf{A} \in \mathbb{R}^{n \times n} .
$$

Usually the state $\mathbf{x}(t)$ is approximated in a lower dimensional space of dimension $r \ll n$, spanned by basis vectors which we subsume in $\mathbf{V}=\left(\mathbf{v}_{1}, \ldots, \mathbf{v}_{r}\right) \in \mathbb{R}^{n \times r}$ :

$$
\mathbf{x}(t) \approx \mathbf{V} \mathbf{z}(t), \quad \text { with } \mathbf{z}(t) \in \mathbb{R}^{r} .
$$

The reduced state $\mathbf{z}$, i.e., the coefficients of the expansion in the reduced space, is defined by a reduced dynamical system that arises from projecting (3) on a test space spanned by the columns of $\mathbf{W}$. There, $\mathbf{W}$ and $\mathbf{V}$ are chosen, such that their columns are biorthonormal, i.e., $\mathbf{W}^{T} \mathbf{V}=\mathbf{I}_{r \times r}$. The Galerkin projection ${ }^{1}$ yields

$$
\tilde{\mathbf{E}} \frac{d}{d t} \mathbf{z}(t)+\tilde{\mathbf{A}} \mathbf{z}(t)+\tilde{\mathbf{B}} \mathbf{u}(t)=0
$$

\footnotetext{
${ }^{1}$ Most frequently $\mathbf{V}$ is constructed to be orthogonal, such that $\mathbf{W}=\mathbf{V}$ can be chosen.
} 
with $\tilde{\mathbf{E}}=\mathbf{W}^{T} \mathbf{E V}, \tilde{\mathbf{A}}=\mathbf{W}^{T} \mathbf{A V} \in \mathbb{R}^{r \times r}$ and $\tilde{\mathbf{B}}=\mathbf{W}^{T} \mathbf{B} \in \mathbb{R}^{r \times m}$. The system matrices $\tilde{\mathbf{E}}, \tilde{\mathbf{A}}, \tilde{\mathbf{B}}$ of this reduced substitute model are of smaller dimension and fixed, i.e., need to be computed only once. However, $\tilde{\mathbf{E}}, \tilde{\mathbf{A}}$ are usually dense whereas the system matrices $\mathbf{E}$ and $\mathbf{A}$ are usually very sparse.

Applying the same technique directly to the nonlinear system means obtaining the reduced formulation (2) by defining $\tilde{\mathbf{q}}(\mathbf{z})=\mathbf{W}^{T} \mathbf{q}(\mathbf{V z})$ and $\tilde{\mathbf{j}}(\mathbf{z})=\mathbf{W}^{T} \mathbf{j}(\mathbf{V z})$. Clearly, $\tilde{\mathbf{q}}$ and $\tilde{\mathbf{j}}$ map from $\mathbb{R}^{r}$ to $\mathbb{R}^{r}$.

To solve network problems of type (2) numerically, usually multistep methods are used. This means that at each timepoint $t_{l}$ a nonlinear equation

$$
\alpha \tilde{\mathbf{q}}\left(\mathbf{z}_{l}\right)+\tilde{\beta}+\tilde{\mathbf{j}}\left(\mathbf{z}_{l}\right)+\tilde{\mathbf{B}} \mathbf{u}\left(t_{l}\right)=\mathbf{0}
$$

has to be solved for $\mathbf{z}_{l}$ which is the approximation of $\mathbf{z}\left(t_{l}\right)$. In the above equation $\alpha$ is the integration coefficient of the method and $\tilde{\beta} \in \mathbb{R}^{r}$ contains history from previous timesteps. Newton techniques that are used to solve (6) usually require an update of the system's Jacobian matrix in each iterations $v$ :

$$
\tilde{\mathbf{J}}_{l}^{(v)}=\left.\left(\alpha \frac{\partial \tilde{\mathbf{q}}}{\partial \mathbf{z}}+\frac{\partial \tilde{\mathbf{j}}}{\partial \mathbf{z}}\right)\right|_{\mathbf{z}=\mathbf{z}_{l}^{(v)}}=\left.\mathbf{W}^{T}\left[\alpha \frac{\partial \mathbf{q}}{\partial \mathbf{x}}+\frac{\partial \mathbf{j}}{\partial \mathbf{x}}\right]\right|_{\mathbf{x}^{(v)}=\mathbf{V} \mathbf{z}_{l}^{(v)}} \mathbf{V}
$$

The evaluation of the reduced system, i.e., $\tilde{\mathbf{q}}$ and $\tilde{\mathbf{j}}$, necessitates in each step the back projection of the $\operatorname{argument} \mathbf{z}$ to its counterpart $\mathbf{V z}$ followed by the evaluation of the full system $\mathbf{q}$ and $\mathbf{j}$ and the projection to the reduced space with $\mathbf{W}$ and $\mathbf{V}$.

Consequently, with respect to computation time no reduction will be obtained unless additional measures are taken or other strategies are pursued.

Up to now, approaches based on linearisation, especially the approach of trajectory piecewise linearisation (TPWL) $[4,5]$, and projection methods based on the Proper Orthogonal Decomposition (POD) are popular. In the following we concentrate on POD and discuss adaptions.

\section{Proper orthogonal decomposition and adaptions}

The POD method, also known as the principal component analysis and KarhunenLoève expansion, provides a technique for analysing multidimensional data [6-8].

POD sets work on data extracted from a benchmark simulation. In a finite dimensional setup like it is given by (1), $K$ snapshots of the state $\mathbf{x}(t)$, the system is in during the training interval $\left[t_{0}, t_{e}\right]$, are collected in a snapshot matrix

$$
\mathbf{X}=\left(\mathbf{x}_{1}, \ldots, \mathbf{x}_{K}\right) \in \mathbb{R}^{n \times K} .
$$

The snapshots, i.e., the columns of $\mathbf{X}$, span a space of dimension $k \leq K$. We search for an orthonormal basis $\left\{\mathbf{v}_{1}, \ldots, \mathbf{v}_{k}\right\}$ of this space that is optimal in the sense that the time-averaged error that is made when the snapshots are expanded in the space spanned by just $r<k$ basis vectors to $\tilde{\mathbf{x}}_{r, i}$, 


$$
\left\langle\left\|\mathbf{x}-\tilde{\mathbf{x}}_{r}\right\|_{2}^{2}\right\rangle \quad \text { with the averaging operator } \quad\langle\mathbf{f}\rangle=\frac{1}{K} \sum_{i=1}^{K} \mathbf{f}_{i}
$$

is minimised. This least squares problem is solved by computing the eigenvalue decomposition of the state covariance matrix $\frac{1}{K} \mathbf{X X}^{T}$ or, equivalently by the singular value decomposition (SVD) of the snapshot matrix (assuming $K>n$ )

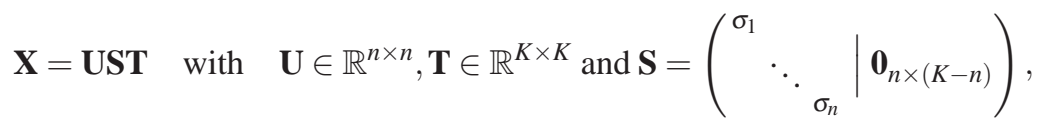

where $\mathbf{U}$ and $\mathbf{T}$ are orthogonal and the singular values statisfy $\sigma_{1} \geq \sigma_{2} \geq \cdots \sigma_{n} \geq 0$. The matrix $\mathbf{V} \in \mathbb{R}^{n \times r}$ whose columns span the reduced subspace is now build from the first $r$ columns of $\mathbf{U}$, where the truncation $r$ is chosen such that

$$
1-\frac{\sum_{i=1}^{n} \sigma_{i}^{2}}{\sum_{i=1}^{r} \sigma_{i}^{2}} \leq \text { tol. }
$$

For the, in this way constructed matrix, it holds $\mathbf{V}^{T} \mathbf{V}=\mathbf{I}_{r \times r}$. Therefore, Galerkin projection as described above can be applied to create a reduced system (2).

For a more detailed introduction to POD in MOR we refer to [9]. For further studies we point to [8] which addresses error analysis for the MOR with POD and [10] where the connection of POD to balanced model reduction can be found.

In the following we reflect two adaptions of POD to overcome the problems that occur in MOR for nonlinear problems and where described in Sec. 2.

\subsection{Missing point estimation}

The missing point estimation (MPE) was proposed in [11] to reduce the cost of updating system information in the solution process of time varying systems arising in computational fluid dynamics. In [12] the MPE approach was brought forward to circuit simulation.

Here, once a POD basis is found, such that (4) holds, there is no Galerkin projection applied. Instead a numerical integration scheme is applied which in general leads to system of $n$ nonlinear equations, analogue to (6), for the $r$ dimensional unkown z. In MPE this system is reduced to dimension $g$ with $r \leq g<n$ by discarding $n-g$ equations. Formally this can be described by multiplying the system with a selection matrix ${ }^{2} \mathbf{P}_{g} \in\{0,1\}^{g \times n}$, stating a $g$-dimensional overdetermined problem

$$
\alpha \mathbf{P}_{g} \mathbf{q}\left(\mathbf{V} \mathbf{z}_{l}\right)+\mathbf{P}_{g} \beta+\mathbf{P}_{g} \mathbf{j}\left(\mathbf{V} \mathbf{z}_{l}\right)+\mathbf{P}_{g} \mathbf{B u}\left(t_{l}\right)=\mathbf{0},
$$

\footnotetext{
2 This means, the matrix has exactly one non-zero entry per row.
} 
which is solved at each timepoint $t_{l}$ for $\mathbf{z}_{l}$ in the least-squares sense [12]. The benefit is that due to the structure of $\mathbf{P}_{g}$ not the full nonlinear functions $\mathbf{q}, \mathbf{j}$ have to be evaluated but just $g$ components.

The choice of $\mathbf{P}_{g}$ is motivated by identifying the $g$ most dominant state variables, i.e., components of $\mathbf{x}$. In terms of the POD basis this is connected to restricting the orthogonal $\mathbf{V}$ to $\tilde{\mathbf{V}}=\mathbf{P}_{g} \mathbf{V} \in \mathbb{R}^{g \times r}$ in an optimal way. This in turn goes down to minimising

$$
\left\|\left(\tilde{\mathbf{V}}^{T} \tilde{\mathbf{V}}\right)^{-1}-\mathbf{I}_{r \times r}\right\| .
$$

Details on reasoning and solving (13) can be found in $[13,14]$

\subsection{Adapted POD}

We put a new approach up for discussion that combines the Galerkin projection with the MPE method. Like described in Sec. 3 we collect snapshots in $\mathbf{X}$ on which we apply an SVD (10). Then we define the matrix $\mathbf{L}=\mathbf{U} \Sigma \in \mathbb{R}^{n \times n}$, with $\Sigma=\operatorname{diag}\left(\sigma_{1}, \ldots, \sigma_{n}\right)$, i.e., we first scale the left-singular vectors with the corresponding singular values. Next we transform the original system (1) by writing $\mathbf{x}(t)=\mathbf{L w}(t)$ and using Galerkin projection:

$$
\frac{d}{d t}\left[\mathbf{L}^{T} \mathbf{q}(\mathbf{L w}(t))\right]+\mathbf{L}^{T} \mathbf{j}(\mathbf{L w}(t))+\mathbf{L}^{T} \mathbf{B u}(t)=\mathbf{0}
$$

Now, we identify separately the $r$ and $g$ most dominant columns of $\mathbf{L}$ and $\mathbf{L}^{T}$, respectively, where the predominance of a column vector $v \in \mathbb{R}^{n}$ is determined by its 2-norm $\|v\|_{2}$. Note that this selection is directly connected to the singular values, i.e., if they decrease rapidly we can expect $r$ and $g$ to be small. We use this information to approximate $\mathbf{L}$ and $\mathbf{L}^{T}$ by matrices that agree with the respective matrix in the selected $r$ and $g$ selected columns but have the $n-r$ and $n-g$ remaining columns set to $\mathbf{0} \in \mathbb{R}^{n}$, respectively. Again, formally this can be expressed with the help of selection matrices $\mathbf{P}_{r} \in\{0,1\}^{r \times n}$ and $\mathbf{P}_{g} \in\{0,1\}^{g \times n}$, respectively:

$$
\mathbf{L} \approx \mathbf{L} \mathbf{P}_{r}^{T} \mathbf{P}_{r} \quad \text { and } \quad \mathbf{L}^{T} \approx \mathbf{L}^{T} \mathbf{P}_{g}^{T} \mathbf{P}_{g}
$$

From this we conclude $\mathbf{L}^{T} \approx \mathbf{P}_{r}^{T} \mathbf{P}_{r} \mathbf{L}^{T} \mathbf{P}_{g}^{T} \mathbf{P}_{g}$. We insert these approximiations in (14) and multiply with $\mathbf{P}_{r}$, bearing in mind that $\mathbf{P}_{r} \mathbf{P}_{r}^{T}=\mathbf{I}_{r \times r}$ :

$$
\frac{d}{d t}\left[\mathbf{P}_{r} \mathbf{L}^{T} \mathbf{P}_{g}^{T} \mathbf{P}_{g} \mathbf{q}\left(\mathbf{L} \mathbf{P}_{r}^{T} \mathbf{P}_{r} \tilde{\mathbf{w}}\right)\right]+\mathbf{P}_{r} \mathbf{L}^{T} \mathbf{P}_{g}^{T} \mathbf{P}_{g} \mathbf{j}\left(\mathbf{L} \mathbf{P}_{r}^{T} \mathbf{P}_{r} \tilde{\mathbf{w}}\right)+\mathbf{P}_{r}^{T} \mathbf{L}^{T} \mathbf{B u}=\mathbf{0} .
$$

Note that due to the approximations to $\mathbf{L}$ and $\mathbf{L}^{T}$ in the above equation $\mathbf{w}$ has changed to $\tilde{\mathbf{w}}$ which can merely be an approximation to the former. We introduce $\mathbf{S}_{r}=\operatorname{diag}\left(\sigma_{1}, \ldots, \sigma_{r}\right)$ and keep the first $r$ columns of $\mathbf{U}$ in $\mathbf{V} \in \mathbb{R}^{n \times r}$. In this way we express $\mathbf{L} \mathbf{P}_{r}^{T}=\mathbf{V} \mathbf{S}_{r}$. Finally we scale (16) with $\mathbf{S}_{r}^{-1}$ and introduce a new unkown 
$\mathbf{z}=\mathbf{S}_{r} \mathbf{P}_{r} \tilde{\mathbf{w}} \in \mathbb{R}^{r}$ from which we can reconstruct the full state by approximation $\mathbf{x} \approx \mathbf{V z}$. We end up with

$$
\frac{d}{d t}\left[\mathbf{W}_{r, g} \mathbf{P}_{g} \mathbf{q}(\mathbf{V z})\right]+\mathbf{W}_{r, g} \mathbf{P}_{g} \mathbf{j}(\mathbf{V z})+\tilde{\mathbf{B}} \mathbf{u}(t)=\mathbf{0},
$$

with $\mathbf{W}_{r, g}=\mathbf{V}^{T} \mathbf{P}_{g}^{T} \in \mathbb{R}^{r \times g}$ and $\tilde{\mathbf{B}}=\mathbf{V}^{T} \mathbf{B}$. Like in the MPE approach just $g$ components of the nonlinear function $\mathbf{q}$ and $\mathbf{j}$ have to be evaluated.

\section{Numerical results}

We consider the academic diode chain model shown in Fig. 1 with 300 nodes. The current traversing a diode with potential $V_{a}$ and $V_{b}$ at the input- and output-node, respectively is described by the nonlinear equation

$$
q\left(V_{a}, V_{b}\right)= \begin{cases}I_{s}\left(e^{\frac{V_{a}-V_{b}}{V_{T}}}-1\right) & \text { if } V_{a}-V_{b}>0.5 \\ 0 & \text { otherwise }\end{cases}
$$

with treshold voltage $V_{T}=0.0256 \mathrm{~V}$ and static current $I_{S}=10^{-14} \mathrm{~A}$. The resistors and capacitors have uniform size $R=10 \mathrm{k} \Omega$ and $C=1 \mathrm{pF}$.

Fig. 1 Diode chain

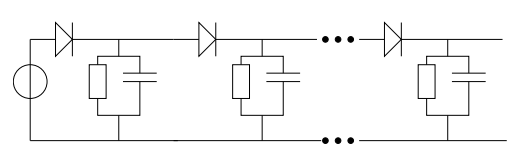

The voltage source defines the input $u(t)$. For the model extraction we choose the step given by

$$
u(t)= \begin{cases}20 & \text { if } t \leq 10 \mathrm{~ns} \\ 170-15 \cdot 10^{9} \cdot t & \text { if } 10 \mathrm{~ns}<t \leq 11 \mathrm{~ns} \\ 5 & \text { if } t>11 \mathrm{~ns}\end{cases}
$$

As Fig. 2 shows, the signal dies out very quickly and just the first 30 diodes operate. This reflects also in the singular values which drop very rapidly. Therefore, for extracting a reduced order model we start the algorithm with the parameters $r=30$ and $g=35$, i.e., the state space is reduced to dimension 30 and the nonlinear functions are downsized to dimension 35.

Of special interest is how a reduced substitute model behaves when signals different to the training signal are applied. For testing purposes we choose

$$
\bar{u}_{1}(t)=7.5 \cos \left(\frac{2 \pi t}{60 \cdot 10^{-9}}\right)+12.5 \text { and } \quad \bar{u}_{2}(t)=9.5 \cos \left(\frac{2 \pi t}{60 \cdot 10^{-9}}\right)+12.5 \text {. }
$$



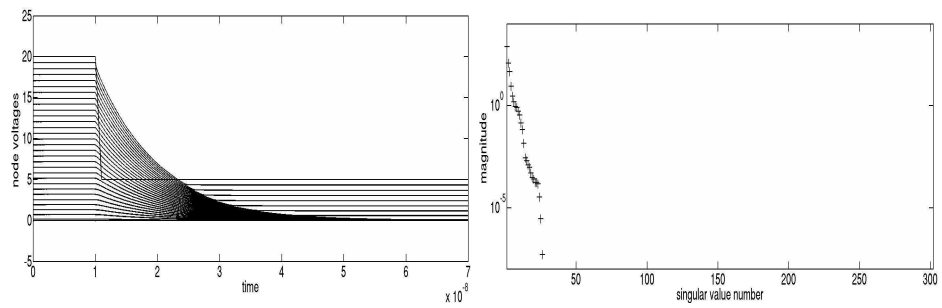

Fig. 2 Diode chain: system's response (left) and singular values (right)

Note that the maximum of $\bar{u}_{1}(t)$ is less than the maximum of the signal $u(t)$ applied for training, whereas $\bar{u}_{2}$ exceeds $u(t)$.

Figure 3 shows the voltages of different nodes as they were produced by solving both the full and the reduced nonlinear system. With the reduced model we were able to accurately reproduce the behaviour of the full system when $\bar{u}_{1}(t)$ was taken as the input. From Table 1 we see that we also achieved a high speedup. Here we also see that the classical POD, i.e, the combination with direct Galerkin projection may even cause more computational work. But, considering the trajectory that was produced with $\bar{u}_{2}(t)$, we see one of the limitations. An explanation might be that the energy in the system during resimulation was higher than during training and extraction. Similar statements can be found in [15] with respect to TPWL.
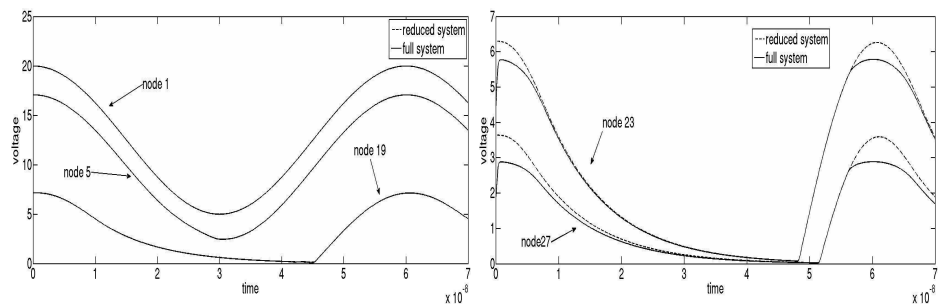

Fig. 3 Resimulation with differing input signal $\bar{u}_{1}(t)$ and $\bar{u}_{2}(t)$.

Table 1 Comparison of cpu time [s]

\begin{tabular}{llll}
\hline input & full & \multicolumn{2}{l}{ classical POD adapted POD } \\
\hline like training & 42.01 & 35.51 & 5.12 \\
$7.5 \cos \ldots$ & 40.22 & 45.34 & 6.28 \\
\hline
\end{tabular}




\section{Conclusion and outlook}

In this paper we study reduced order modelling of nonlinear IC models. We review the problems that show up when MOR techniques for linear problems are applied to nonlinear systems. These problems arise from the necessity to still evaluate the full nonlinear system. To this point ways to overcome the problem are to either linearise the nonlinear system and apply MOR to the arising linear systems, like done in TPWL, or to adapt projection methods, like done in MPE in connection with POD. We introduce a new adaption of the latter approach. Put to test with an academic example it shows nice results, especially with input signals that differ from training signals. However, the new approach has to be studied more carefully regarding its general applicability.

Acknowledgements The work presented is funded by the Marie-Curie Transfer-of-Knowledge project O-MOORE-NICE!

\section{References}

1. Antoulas, A.C.: Approximation of Large-Scale Dynamical Systems. SIAM (2005)

2. Scherpen, J.M.A.: Balancing for nonlinear systems. Ph.D. thesis, University of Twente (1994)

3. Ionescu, T.C., Scherpen, J.M.A.: Positive Real Balancing for Nonlinear Systems. In: G. Ciuprina, D. Ioan (eds.) Scientific Computing in Electrical Engineering - SCEE 2006, Mathematics in Industry, vol. 11, pp. 153-159. The European Consortium for Mathematics in Industry, Springer-Verlag Berlin Heidelberg (2007)

4. Rewieński, M.J., White, J.: A trajectory piecewise-linear approach to model order reduction and fast simulation of nonlinear circuits and micromachined devices. IEEE Trans. CAD Int. Circ. Syst. 22(2), 155-170 (2003)

5. Voß, T., Pulch, R., ter Maten, J., El Guennouni, A.: Trajector piecewise linear aproach for nonlinear differential-algebraic equations in circuit simulation. In: G. Ciuprina, D. Ioan (eds.) Scientific Computing in Electrical Engineering - SCEE 2006, pp. 167-173. Springer (2007)

6. Holmes, P., Lumley, J., Berkooz, G.: Turbulence, Coherent Structures, Dynamical Systems and Symmetry. Cambrige University Press, Cambrige, UK (1996)

7. Loève, M.: Probability Theory. Van Nostrand (1955)

8. Rathinam, M., Petzold, L.R.: A new look at proper orthogonal decomposition. SIAM J. Numer. Anal. 41(5), 1893-1925 (2003)

9. Pinnau, R.: Model reduction via proper orthogonal decomposition. In: W. Schilders, H. van der Vorst, J. Rommes (eds.) Model order reduction: theory, applications, and research aspects, pp. 95-109. Springer (2008)

10. Willcox, K., Peraire, J.: Balanced model reduction via the proper orthogonal decomposition. AIAA Journal 40(11), 2323-2330 (2002)

11. Astrid, P.: Reduction of process simulation models: a proper orthogonal decomposition approach. Ph.D. thesis, Technische Universiteit Eindhoven (2004)

12. Astrid, P., Verhoeven, A.: Application of least squares mpe technique in the reduced order modeling of electrical circuits. In: Proceedings of the 17th Int. Symp. MTNS, pp. 1980-1986 (2006)

13. Astrid, P., Weiland, S.: On the construction of pod models from partial observations. In: Proceedings of the 44rd IEEE Conference on Decision and Control, pp. 2272-2277 (2005)

14. Verhoeven, A.: Redundancy reduction of ic models by multirate time-integration and model order reduction. Ph.D. thesis, Technische Universiteit Eindhoven (2008)

15. Rewieński, M.J.: A trajectory piecewise-linear approach to model order reduction of nonlinear dynamical systems. Ph.D. thesis, Massachusetts Institute of Technology (2003) 
PREVIOUS PUBLICATIONS IN THIS SERIES:

\begin{tabular}{|c|c|c|c|}
\hline Number & Author(s) & Title & Month \\
\hline $09-17$ & $\begin{array}{l}\text { M.A. Etaati } \\
\text { R.M.M. Mattheij } \\
\text { A.S. Tijsseling } \\
\text { A.T.A.M. de Waele }\end{array}$ & $\begin{array}{l}\text { One-dimensional simulation } \\
\text { of a stirling three-stage pulse- } \\
\text { tube refrigerator }\end{array}$ & May '09 \\
\hline 09-18 & $\begin{array}{l}\text { R. Duits } \\
\text { E. Franken }\end{array}$ & $\begin{array}{l}\text { Left-invariant diffusions on } \\
\mathrm{R}^{3} \times \mathrm{S}^{2} \text { and their application } \\
\text { to crossing-preserving } \\
\text { smoothing of HARDI-images }\end{array}$ & May ‘09 \\
\hline 09-19 & $\begin{array}{l}\text { T. Bechtold } \\
\text { M. Striebel } \\
\text { K. Mohaghegh } \\
\text { E.J.W. ter Maten }\end{array}$ & $\begin{array}{l}\text { Nonlinear model order } \\
\text { reduction in nanoelectronics: } \\
\text { combination of POD and } \\
\text { TPWL }\end{array}$ & June ‘09 \\
\hline $09-20$ & $\begin{array}{l}\text { K. Mohaghegh } \\
\text { R. Pulch } \\
\text { M. Striebel } \\
\text { E.J.W. ter Maten }\end{array}$ & $\begin{array}{l}\text { Model order reduction for } \\
\text { semi-explicit systems of } \\
\text { differential algebraic } \\
\text { equations }\end{array}$ & June '09 \\
\hline $09-21$ & $\begin{array}{l}\text { A. Verhoeven } \\
\text { M. Striebel } \\
\text { E.J.W. ter Maten }\end{array}$ & $\begin{array}{l}\text { Model order reduction for } \\
\text { nonlinear IC models with } \\
\text { POD }\end{array}$ & June '09 \\
\hline
\end{tabular}

